\title{
Real-Time System Identification of a Nonlinear Four-Story Steel Frame Structure-Application to Structural Health Monitoring
}

\author{
Christopher E. Hann, Ishan Singh-Levett, Bruce L. Deam, John B. Mander, and J. Geoffrey Chase
}

\begin{abstract}
Structural health monitoring (SHM) is a means of identifying damage from structural response to environmental loads. Real-time SHM is of particular use for rapid assessment of structural safety by owners and civil defense authorities. This paper presents an algorithm for real-time SHM during earthquake events using only acceleration measurements and infrequently measured displacement motivated by global positioning system. The algorithm identifies a nonlinear baseline model including hysteretic dynamics and permanent deformation using convex integral-based fitting methods and piecewise linear least squares fitting. The methodology identifies pre and postyield stiffness, elastic and plastic components of displacement, and final residual displacement. It thus identifies key measures of damage affecting the immediate safety or use of the structure and the long-term cost of repair and retrofit. The algorithm is tested with simulated response data using the El-Centro earthquake record and with measured response data. Both data sets are based on a four-story nonlinear steel frame structure using the El-Centro ground motion record. Overall, the algorithm is shown to provide accurate indications of the existence, location, and magnitude of structural damage for nonlinear shear-type buildings. Additionally, the identified permanent displacement is a particularly useful damage measure for the construction of probabilistic fragility functions.
\end{abstract}

Index Terms-Model-based parameter identification, permanent displacement, real-time algorithm, structural health monitoring (SHM).

\section{INTRODUCTION}

$\mathbf{S}$ TRUCTURAL health monitoring (SHM) is the process of comparing the current state of a structure's condition relative to a baseline state using measured response data and determining the existence, location, and degree of damage that may

Manuscript received October 04, 2008; revised December 17, 2008; accepted December 17, 2008. First published August 18, 2009; current version published September 23, 2009. The associate editor coordinating the review of this paper and approving it for publication was Dr. Subhas Mukhopadhyay.

C. E. Hann and J. G. Chase are with the Department of Mechanical Engineering, University of Canterbury, Christchurch 8140, New Zealand (e-mail: Chris.Hann@canterbury.ac.nz; Geoff.Chase@ canterbury.ac.nz).

B. L. Deam is with the Department of Civil Engineering, University of Canterbury, Christchurch 8140, New Zealand (e-mail: Bruce.Deam@ canterbury.ac. $\mathrm{nz})$.

J. B. Mander is with the Zachry Department of Civil Engineering, Texas A\&M University, College Station, TX 77843 USA (e-mail: jmander@civil. tamu.edu).

I. Singh-Levett is with the HGL Dynamics Ltd., Godalming GU7 1HZ, U.K. (e-mail: isinghlevett@hgl-dynamics.com).

Color versions of one or more of the figures in this paper are available online at http://ieeexplore.ieee.org.

Digital Object Identifier 10.1109/JSEN.2009.2022434 exist, particularly after a damaging input, such as an earthquake or other large environmental load. Many current vibration-based SHM methods, particularly for large civil structures, are based on modal parameter damage detection in both the time series and frequency domain [1]. These frequency methods can also simplify typical procedures of visual or localized experimental methods, such as acoustic or ultrasonic methods [2], magnetic field methods, radiography, eddy-current methods, or thermal field methods [3], as they do not require visual inspection of the structure.

Current modal methods are more applicable to steel frame and bridge structures, where vibration response is more linear [1], [3], [4]. Additionally, many existing algorithms assume that data sets from the undamaged structure are available [2], [5].

A SHM method must identify localized damage, be robust in the presence of noise, and evaluate structural health rapidly or in real time [6]. Most existing methods that could potentially provide real-time SHM are eigensystem realization algorithm-based methods. These methods typically only use accelerometers as sensors and rely on the change in natural frequencies to detect damage [7]. However, a change in a frequency does not necessarily represent damage, particularly with highly nonlinear responses [8], [9]. Significant changes in story stiffnesses are often required as well, which would normally cause clearly visible damage [9].

Chase et al. [4] identified changes in structural stiffness in real-time using least mean square-based adaptive filtering. However, this method requires measurement of velocity and displacement, which is impractical in many realistic cases due to excessive sensor requirements.

Recently, there have been significant advances in global positioning system (GPS) displacement monitoring technology for large structures [9], [10]. Displacements can be measured with 1-3 mm accuracy for up to $3-4 \mathrm{~Hz}$ in the modal frequencies of the rigid structure [10], and for $1 \mathrm{~Hz}$, measurement errors have been stated as less than $12 \%$ [11]. The use of GPS opens up new opportunities in real-time SHM [9].

The approach in this paper combines the relatively low-resolution GPS-measured displacements with high-resolution accelerometers. The method utilizes a general model structure that is loosely based on the nonlinear Bouc-Wen Hysteresis model [12]. However, the method can directly identify the permanent displacement and time-varying stiffness of multistory buildings independent of any specific hysteresis model. 
By carefully reformulating the differential equation model in terms of integrals of the measured data, a highly nonlinear optimization is transformed into a standard linear least squares problem. The solution also uses a novel displacement correction method that accounts for drift and modeling error. The end result is a computationally fast and accurate model-based identification method, which can detect important structural changes, such as permanent deformation. The method can be readily implemented in real time, is robust in the presence of noise, and shows the potential to accurately identify localized damage.

\section{Methodology}

\section{A. Nonlinear Baseline Structure Model}

The Bouc-Wen model [12] is commonly used in seismic engineering to model hysteresis, e.g., [13] and is given by the matrix equation defined as follows:

$$
\begin{aligned}
M \ddot{x}(t)+C \dot{x}(t)+r(x, z) & =-M \ddot{x}_{g}(t) \\
r(x, z) & =K_{e} x(t)+K_{h} z(t) \\
K_{e}=\alpha K_{p} x(t), \quad K_{h} & =(1-\alpha) K_{p}
\end{aligned}
$$

where $M$ is the mass matrix, $C$ is the viscous damping matrix, $K_{e}$ and $K_{h}$ are elastic and hysteric stiffnesses, $K_{p}$ is the preyield stiffness coefficient, $0 \leq \alpha \leq 1$ is the bilinear factor, $x(t)$ is the displacement vector, $\dot{x}(t)$ is the velocity vector, $\ddot{x}(t)$ is the acceleration vector, and $\ddot{x}_{g}(t)$ is the ground acceleration vector. The cases of $\alpha=0$ and $\alpha=1$ correspond to the restoring force $r(x, z)$ in (2) being either purely hysteretic or elastic, respectively. The vector $z(t)$ represents hysteretic displacement. For purposes of testing in simulation, the Bouc-Wen hysteresis is defined [12] as follows:

$$
\begin{array}{r}
\dot{z}_{i}(t)=\dot{r}_{i}(t)\left[1-0.5\left[1+\operatorname{sgn}\left(\dot{r}_{i}(t) z_{i}(t)\right)\right]\left|\frac{z_{i}(t)}{Y_{i}}\right|^{n_{i}}\right] \\
i=1, \ldots, \bar{N}
\end{array}
$$

where $\dot{r}_{i}(t)$ is the velocity of story $i$ relative to story $i-1, \bar{N}$ is the number of degrees of freedom (DF), $Y$ is the yield displacement, and $n_{i}$ is a shaping parameter determining the curve from elastic to plastic force-deflection behavior. Permanent displacement of an element is defined as

$$
D_{i}(t)=\frac{r_{i}(t)-z_{i}(t)}{\left[1+\frac{\alpha_{i}}{1-\alpha_{i}}\right]}
$$

where $D_{i}(t)$ is the permanent displacement of story $i$ and $r_{i}(t)$ is the displacement of story $i$ relative to story $i-1$

$$
r_{i}(t)=x_{i}(t)-x_{i-1}(t)
$$

In the identification method, $M$ and $C$ are assumed to be reasonably well-known and constant. Therefore, the variables to be identified are preyield stiffness $K_{p}$ and the permanent displacement $D(t)$. All other values are estimated or known.

\section{B. Displacement and Velocity Estimation}

Identification of $K_{p}$ and $D(t)$ from (1) and (5) requires knowledge of the ground acceleration and the structural displacement, velocity, and acceleration. Integrated estimates of displacement and velocity are naturally subject to drift and numerical error. However, with the availability of GPS [9]-[11], this error can be corrected using displacement data measured at relatively low frequency. The displacement measurement was taken at $1 \mathrm{~Hz}$ with $10 \%$ measurement noise, which is based on errors in [9]-[11].

Integrated displacement and velocity are defined as follows:

$$
\begin{aligned}
& x_{k}(t)=\int_{0}^{t}\left[\int_{0}^{t} \ddot{x}_{k} d t\right] d t \\
& \dot{x}_{k}(t)=\int_{0}^{t} \ddot{x}_{k}(t) d t
\end{aligned}
$$

where $\ddot{x}_{k}(t)$ is the $1 \mathrm{kHz}$ sampled acceleration data for the $k$ th floor and the integrals refer to numerical integration. The integration displacement error to be corrected by the measured $1 \mathrm{~Hz}$ displacement $x_{\text {meas }, k}$ is defined as

$$
e_{i}=x_{\text {meas }, k}(h i)-x(h i), \quad i=1, \ldots, N
$$

where $e_{i}$ is the error at the time $t=h i$ and $h$ is the time interval at which displacement measurements are reported. Note that $h=1$ for the case where displacement is measured at $1 \mathrm{~Hz}$.

The measured and integrated displacements $x_{\text {meas }, k}$ and $x_{k}$ are given by (7) and (8), respectively. The velocity error $c_{i}$ can be calculated by simple numerical differentiation of $e_{i}$

$$
c_{i}=\frac{\left(e_{i}-e_{i-1}\right)}{h}, \quad i=1, \ldots, N
$$

where $e_{0}$ and $c_{0}$ are both assumed to be zero.

The displacement error $e_{i}, i=1, \ldots N$ of (10) is then linearly interpolated using a set of piecewise $\mathrm{C}^{1}$ cubics

$$
\begin{aligned}
\bar{f}(t)= & R_{i}(t), \quad h(i-1)<t<h i, \quad i=1, \ldots, N \\
R_{i}(t)= & a_{i 1}[t-h(i-1)]^{3}+a_{i 2}[t-h(i-1)]^{2} \\
& +a_{i 3}[t-h(i-1)]+a_{i 4}, \quad h(i-1) t<t<h i
\end{aligned}
$$

where $\left[\begin{array}{llll}a_{i 1} & a_{i 2} & a_{i 3} & a_{i 4}\end{array}\right], i=1, \ldots, N$ are recursively defined as

$$
\begin{aligned}
& {\left[\begin{array}{cccc}
h^{3} & h^{2} & h & 1 \\
3 h^{2} & 2 h & 1 & 0 \\
0 & 0 & 1 & 0 \\
0 & 0 & 0 & 1
\end{array}\right]\left\{\begin{array}{l}
a_{i 1} \\
a_{i 2} \\
a_{i 3} \\
a_{i 4}
\end{array}\right\}} \\
& =\left\{\begin{array}{c}
e_{i} \\
c_{i} \\
3 a_{i-1,1}(h i)^{2}+2 a_{i-1,2}(h i)+a_{i-1,3} \\
a_{i-1,1}(h i)^{3}+a_{i-1,2}(h i)^{2}+a_{i-1,3}(h i)+a_{i-1,4}
\end{array}\right\}
\end{aligned}
$$

and $h=1$ is the length of the time interval of interest. Note that (11) satisfies

$$
\begin{gathered}
\bar{f}(h i)=R_{i}(h i)=e_{i} \\
\bar{f}^{\prime}(h i)=R_{i}^{\prime}(h i)=c_{i}
\end{gathered}
$$


The time-varying corrected displacement may now be estimated by adding the integrated displacement and the continuous corrector function $\bar{f}(t)$ of $(11)$

$$
d(t)=\int_{0}^{t}\left[\int_{0}^{t} \ddot{x}_{k} d t\right] d t+\bar{f}(t),(i-1)<t<i, \quad i=1, \ldots, N .
$$

The time-varying corrected velocity and acceleration are then calculated by numerical differentiation as follows:

$$
\begin{aligned}
& v\left(t_{i}\right)=\frac{d\left(t_{i+1}\right)-d\left(t_{i-1}\right)}{2 \Delta t} \\
& a\left(t_{i}\right)=\frac{v\left(t_{i+1}\right)-v\left(t_{i-1}\right)}{2 \Delta t} .
\end{aligned}
$$

Note that the estimated acceleration used for identification from (17) is, in general, slightly different from the measured acceleration. Simulation has shown that using the directly measured acceleration in parameter identification results in the method going unstable. The reason for the instability is that the measured acceleration is not the double derivative of the estimated displacement, which causes a mismatch in (1). Thus, it is critical to use the estimated acceleration of (17).

\section{Integral-Based System Identification}

The goal is to identify the parameters $K_{p}$ and $D(t)$ from the nonlinear equations of motion in the matrix (1). The identification could be achieved by standard nonlinear least squares optimization. However, for multistory buildings and time-varying stiffness and permanent displacement there are many variables, and a gradient descent would be very computationally expensive.

Therefore, an alternative approach for identifying the model of (1) for a general hysteretic displacement $z(t)$ is considered. First, (1) is written as its constituent components as follows:

$$
\begin{aligned}
& m_{1} \ddot{x}_{1}(t)+\sum_{b=1}^{\bar{N}} c_{1, b} \dot{x}_{b}(t)+k_{e}^{(2)}\left[x_{1}(t)-x_{2}(t)\right] \\
& \quad+k_{e}^{(1)} x_{1}(t)-k_{h}^{(2)} z_{2}(t)+k_{h}^{(1)} z_{1}(t)=-m_{1} \ddot{x}_{g}(t) \\
& m_{\bar{N}} \ddot{x}_{\bar{N}}(t)+\sum_{b=1}^{\bar{N}} c_{\bar{N}, b} \dot{x}_{b}(t)+k_{e}^{(\bar{N})}\left[x_{\bar{N}}(t)-x_{\bar{N}-1}(t)\right] \\
& \quad+k_{h}^{(\bar{N})} z_{\bar{N}}(t)=-m_{\bar{N}} \ddot{x}_{g}(t) \\
& m_{w} \ddot{x}_{w}(t)+\sum_{b=1}^{N} c_{w, b} \dot{x}_{b}(t)+k_{e}^{(w)}\left[x_{w}(t)-x_{w-1}(t)\right] \\
& \quad+k_{e}^{(w+1)}\left[x_{w}(t)-x_{w+1}(t)\right]+k_{h}^{(w)} z_{w}(t) \\
& \quad-k_{h}^{(w+1)} z_{w+1}(t)=-m_{w} \ddot{x}_{g}(t), w=2, \ldots, \bar{N}-1 .
\end{aligned}
$$

Note that superscripts " $(w)$ " are used on the parameters $k_{e}$ and $k_{h}$ and subscripts " $w$ " are used on the rest of the parameters to denote the story number. Therefore, (18) defines the motion of the bottom story, (19) defines the motion of any intermediate story, $w=2, \ldots, \bar{N}-1$ and (20) defines the motion of the top story $\bar{N}$.
Next, a revised equation of motion for the first story can be written by the summation of (18)--(20). The summation of all the left-hand sides of (18)--(20) gives

$$
\begin{aligned}
& \sum_{k=1}^{\bar{N}} m_{k} \ddot{x}_{k}(t)+\sum_{k=1}^{\bar{N}} \sum_{b=1}^{\bar{N}} c_{k, b} \dot{x}_{b}(t) \\
& \quad+\sum_{k=1}^{\bar{N}} k_{e}^{(k)}\left[x_{k}(t)-x_{k-1}(t)\right]+\sum_{k=1}^{\bar{N}} k_{h}^{(k)} z_{k}(t) \\
& \quad-\sum_{k=2}^{\bar{N}} k_{e}^{(k)}\left[x_{k}(t)-x_{k-1}(t)\right]-\sum_{k=2}^{\bar{N}} k_{h}^{(k)} z_{k}(t)
\end{aligned}
$$

where the restoring force terms are

$$
\sum_{k=1}^{\bar{N}} k_{e}^{(k)}\left[x_{k}(t)-x_{k-1}(t)\right]+\sum_{k=1}^{\bar{N}} k_{h}^{(k)} z_{k}(t)
$$

and

$$
-\sum_{k=2}^{\bar{N}} k_{e}^{(k)}\left[x_{k}(t)-x_{k-1}(t)\right]-\sum_{k=2}^{\bar{N}} k_{h}^{(k)} z_{k}(t) .
$$

The terms shown in (22a) and (22b) form a telescoping sum, leaving $k_{e}^{(1)}\left[x_{2}(t)-x_{1}(t)\right]+k_{h}^{(1)}\left[z_{1}(t)\right]$. Thus, the revised equation of motion for the bottom story is written as follows:

$$
\begin{array}{r}
\sum_{k=1}^{\bar{N}} m_{k} \ddot{x}_{k}(t)+\sum_{k=1}^{\bar{N}} \sum_{b=1}^{\bar{N}} c_{k, b} \dot{x}_{b}(t)+k_{e}^{(1)} x_{1}(t)+k_{h}^{(1)} z_{1}(t) \\
=-\sum_{k=1}^{\bar{N}} m_{k} \ddot{x}_{g}(t)
\end{array}
$$

and similarly for any floor $w, w=2, \ldots, \bar{N}-1$

$$
\begin{aligned}
& \sum_{k=w}^{\bar{N}} m_{k} \ddot{x}_{k}(t) \\
& \quad+\sum_{k=w}^{\bar{N}} \sum_{b=1}^{\bar{N}} c_{k, b} \dot{x}_{b}(t)+k_{e}^{(w)}\left[x_{w}(t)-x_{w-1}(t)\right]+k_{h}^{(w)} z_{w}(t) \\
& =-\sum_{k=w}^{\bar{N}} m_{k} \ddot{x}_{g}(t) .
\end{aligned}
$$

The top story $\bar{N}$ is kept in the form given by (21). The summation process eliminates the unknowns $k_{e 2}, k_{h 2}$, and $z_{2}(t)$ from (18) and for each $w$ in (19) eliminates the unknowns $k_{e}^{(w+1)}, k_{h}^{(w+1)}$, and $z_{w+1}(t)$. Also, the only unknowns in (23) are $k_{e}^{(\bar{N})}, k_{h}^{(\bar{N})}$, and $z_{\bar{N}}(t)$. This process ensures that all the unknowns are specific to each story. The estimated acceleration $a_{w}(t)$, velocity $v_{w}(t)$, and displacement $d_{w}(t)$ are then calculated using (15)-(17) to approximate $\ddot{x}_{w}(t), \dot{x}_{w}(t)$, and $x_{w}(t)$, respectively, for (21), (23), and (24). Equations (21), (23), and (24) can then be rewritten as one equation for any story $w$, $w=1, \ldots, \bar{N}$ as follows:

$$
\begin{aligned}
& k_{p}^{(w)} r_{w}(t)+\Delta Z^{(w)}(t) \\
& =-\sum_{k=w}^{\bar{N}} m_{k} \ddot{x}_{g}(t)-\sum_{k=w}^{\bar{N}} m_{k} a_{k}(t)-\sum_{k=w}^{\bar{N}} \sum_{b=1}^{\bar{N}} c_{k, b} v_{b}(t)
\end{aligned}
$$




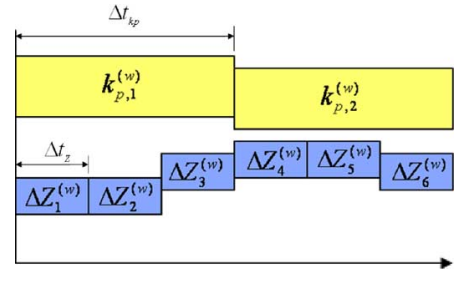

Fig. 1. Variation of fitted parameters for $l=3$.

where

$$
\begin{aligned}
k_{p}^{(w)} & =k_{e}^{(w)}+k_{h}^{(w)}, \quad w=1, \ldots, \bar{N} \\
\Delta Z^{(w)}(t) & =k_{h}^{(w)}\left[z_{w}(t)-r_{w}(t)\right], \quad w=1, \ldots, \bar{N}
\end{aligned}
$$

and $r_{w}(t)$ is the relative displacement of story $w$ as defined in (6).

The parameters $k_{p}^{(w)}$ and $\Delta Z^{(w)}(t)$ can now be found in a piecewise variation for a given identification interval using (25), where the term $r_{w}(t)$ on the left-hand side and all terms on the right-hand side are either measured or estimated.

To be as general as possible and to potentially account for unmodeled dynamics, the parameter $k_{p}^{(w)}$ is made time varying as follows:

$$
k_{p}^{(w)}(t)=k_{p, i}^{(w)}, \quad(i-1) \Delta t_{k p} \leq t \leq i \Delta t_{k p}, \quad i=1, \ldots, N
$$

where $\Delta t_{k p}$ is a user-selected interval over which piecewise constant behavior is a reasonable approximation for the structure considered. The time-varying term $\Delta Z^{w}(t)$ is represented by a piecewise constant function as follows:

$\Delta Z^{w}(t)=\Delta Z_{w j}, \quad(j-1) \Delta t_{Z} \leq t \leq j \Delta t_{Z}, \quad j=1, \ldots, M$.

For ease of fitting $\Delta t_{k p}$ is defined as follows:

$$
\Delta t_{k p}=l \Delta t_{Z}
$$

Thus, $l$ values of $\Delta Z_{j}^{(w)}$ are fitted alongside every single value of $k_{p, i}^{(w)}$ as shown in Fig. 1 for the case $l=3$.

For a given story number $w,(25)$ can now be rewritten as $l$ equations for each interval $\Delta t_{k p}$ corresponding to $i=1, \ldots, N$, as illustrated in Fig. 1

$$
\begin{aligned}
& k_{p, i}^{(w)} r_{w}(t)+\Delta Z_{j}^{(w)}(t) \\
& =-\sum_{k=w}^{\bar{N}} m_{k} \ddot{x}_{g}(t)-\sum_{k=w}^{\bar{N}} m_{k} a_{k}(t)-\sum_{k=w}^{\bar{N}} \sum_{b=1}^{\bar{N}} c_{k, b} v_{b}(t) \\
& \quad j=l(i-1)+1, \ldots, l i, \quad i \in\{1, \ldots, N\} \\
& \quad(j-1) \Delta t_{Z} \leq t \leq j \Delta t_{Z} .
\end{aligned}
$$

Identification of $k_{p}^{(w)}$ and $\Delta Z^{(w)}(t)$ for each story number $w$ requires the construction of a system of linear equations, each in the form of (31). For the example given in Fig. 1, three values of $t$ could be chosen in each time interval $(j-1) \Delta t_{Z} \leq t \leq$ $j \Delta t_{Z}$ of (31). This choice will give nine equations for each time interval $\Delta t_{k p}$ in Fig. 1, and thus, in total $9 N$ linear equations in $4 N$ unknown parameters for each story number $w$. The $4 N$ parameters are defined as follows:

$$
\underline{\alpha}^{(w)}=\left\{\underline{\alpha}_{1}^{(w)}, \ldots, \underline{\alpha}_{N}^{(w)}\right\}^{T}
$$

where

$$
\underline{\alpha}_{i}^{(w)}=\left\{k_{p}^{(w)}, \Delta Z_{3 i-2}^{(w)}, \Delta Z_{3 i-1}^{(w)}, \Delta Z_{3 i}^{(w)}\right\}^{T}, \quad i=1, \ldots, N .
$$

The linear equations for each $w=1, \ldots, \bar{N}$ can be represented as $N$ matrix equations each of the form

$$
A_{w, i} \underline{\alpha}_{i}^{(w)}=b_{w, i}, \quad i=1, \ldots, N .
$$

In this case, the matrix $A_{w, i}$ for (34) is of the form

$$
A_{w, i}=\left[\begin{array}{ccccccccc}
r_{w}\left(t_{i 1}\right) & & & & \cdots & & & & r_{w}\left(t_{i 9}\right) \\
1 & 1 & 1 & 0 & 0 & 0 & 0 & 0 & 0 \\
0 & 0 & 0 & 1 & 1 & 1 & 0 & 0 & 0 \\
0 & 0 & 0 & 0 & 0 & 0 & 1 & 1 & 1
\end{array}\right]^{T}
$$

and the right-hand side vector $b_{w, i}$ is of the form

$$
=\left[\begin{array}{c}
-\sum_{k=q}^{\bar{N}} m_{k} \ddot{x}_{g}\left(t_{i 1}\right)-\sum_{k=w}^{\bar{N}} m_{k} a_{k}\left(t_{i 1}\right)-\sum_{k=w}^{\bar{N}} \sum_{b=1}^{\bar{N}} c_{k, b} v_{b}\left(t_{i 1}\right) \\
-\sum_{k=q}^{\bar{N}} m_{k} \ddot{x}_{g}\left(t_{i 2}\right)-\sum_{k=w}^{\bar{N}} m_{k} a_{k}\left(t_{i 2}\right)-\sum_{k=w}^{\bar{N}} \sum_{b=1}^{\bar{N}} c_{k, b} v_{b}(t 2) \\
\vdots \\
-\sum_{k=q}^{\bar{N}} m_{k} \ddot{x}_{g}\left(t_{i 9}\right)-\sum_{k=w}^{\bar{N}} m_{k} a_{k}\left(t_{i 9}\right)-\sum_{k=w}^{\bar{N}} \sum_{b=1}^{\bar{N}} c_{k, b} v_{b}\left(t_{i 9}\right)
\end{array}\right] .
$$

The least squares solution of the matrix (34) determines the vector $\underline{\alpha}^{(w)}$ of unknowns for each $w=1, \ldots, \bar{N}$. Assuming that the factor $\alpha$ in (2) is known, the parameters $\left\{k_{e, i}^{(w)}, i=\right.$ $\left.1, \ldots, N\},\left\{k_{w, i}^{(w)}, i=1, \ldots N\right\}\right\}$, and $\left\{D_{j}^{(w)}, j=1, \ldots, M\right\}$ can then be found as follows:

$$
\begin{aligned}
k_{e, i}^{(w)} & =\alpha k_{p, i}^{(w)} \\
k_{h, i}^{(w)} & =(1-\alpha) k_{p, i}^{(w)} \\
D_{j}^{(w)} & =\frac{-\Delta Z_{j}^{(w)}}{k_{h, i}^{(w)}\left(1+\frac{\alpha}{1-\alpha}\right)}
\end{aligned}
$$

where $j=l(i-1)+1, \ldots, l i$ and $i=1, \ldots, N$.

Note that $D_{j}^{(w)}$ is the permanent displacement of story $w$ relative to story $w-1$; the absolute permanent displacement of story $w-1$ can be calculated as follows:

$$
D A_{j}^{(w)}=\sum_{k=1}^{w} D_{j}^{(k)}
$$

This process is performed for each story $w$ and each time interval $(i-1) \Delta t_{k p} \leq t \leq i \Delta t_{k p}$ to identify the stiffness and 


\section{Shear Building Fitting Process}

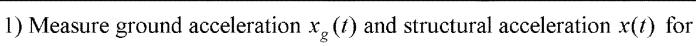

1) Measure ground acceleration $x_{g}(t)$ and structural acceleration $x(t)$ for period of interest $(i-1) \Delta t_{k p} \leq t \leq i \Delta t_{k p}$

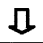

2) Use Equations (7) and (8) to estimate displacement $x(t)$ and velocity $x(t)$

\section{I}

3) Calculate error in $x(t)$ and $x(t)$ using low frequency

displacement measurement $x_{\text {meas }}(t)$ in Equations (9) and (10)

\section{I}

4) Obtain corrected displacement $d(t)$, velocity $v(t)$ and acceleration $a(t)$ using Equations (11)-(17)

\section{II}

5) Create matrix $A_{w, i}$ and vector $b_{w, i}$ for storey $w$ from Equations (35) and (36)

I

6) Solve matrix Equation (34) to find linear stiffness $k_{p, i}^{(w)}$ and permanent displacement parameters $\Delta Z_{j}^{(w)}$

\section{I}

7) Find elastic and hysteretic stiffnesses $k_{e j}^{(w)}$ and $k_{h j}^{(w)}$ permanent displacements $D_{j}^{(w)}$ from Equations (37)-(39)

\section{II}

8) Repeat steps 5-7 for all other storeys

II

9) Repeat steps 1-8 for all other periods of interest

Fig. 2. Flow chart depicting fitting process.

permanent displacement of each story over the entire record. A graphical depiction of the process is shown in Fig. 2.

In practice, $\Delta t_{k p}$ and $\Delta t_{Z}$ might typically be chosen to be approximately $2 \mathrm{~s}$ and $0.2 \mathrm{~s}$, respectively, resulting in a multiple, $l$, of 10 in (31) and $\alpha_{i}$ in (33) of size $11 \times 1$. Since the data can be sampled at $1 \mathrm{kHz}$, for maximum accuracy, all the 2000 data points in each period of $\Delta t_{k p}=2$ are used. Thus, the resulting matrices $A_{w, i}$ are of size $2000 \times 11$ and the vectors $b_{w, i}$ are of size $2000 \times 1$. Using 2000 sets of measured data to estimate 11 parameters ensure that the algorithm is very robust in the presence of noise.

\section{RESUltS AND Discussion}

\section{A. Simulation Structure}

The method given in Fig. 2 with $\Delta t_{k p}=2, \Delta t_{z}=0.4$ is first tested on simulated data using the model of (1) and the Bouc-Wen hysteresis of (4). The output acceleration is sampled at $1 \mathrm{kHz}$ and the output displacement is sampled at $1 \mathrm{~Hz}$. About $10 \%$ random uniformly distributed noise is added to both measurements. Simulations were performed in Matlab.

Each story had a preyield stiffness of $1610 \mathrm{~N} / \mathrm{m}$ and mass of $1 \mathrm{~kg}$, resulting in an undamped fundamental natural period of $0.45 \mathrm{~s}$ for the structure. This period was chosen to closely match the natural period of the laboratory structure, which has been calculated at $0.47 \mathrm{~s}$ [14]. A diagonal mass matrix was used in simulation. The laboratory structure has been calculated to have between $0.9 \%$ and $2.9 \%$ damping, depending on the magnitude of response [14]. Thus, the following damping matrix
TABLE I

FITTED STIFFNESS CONFIDENCE INTERVALS OVER FIRST $30 \mathrm{~s}$ WITH 10\% NOISE

\begin{tabular}{|r|r|r|r|}
\hline Storey & Mean Stiffness (N/m) & $90 \%$ Confidence Maximum & $90 \%$ Confidence Minimum \\
\hline 1 & 1601 & 1628 & 1556 \\
\hline 2 & 1602 & 1635 & 1561 \\
\hline 3 & 1607 & 1645 & 1565 \\
\hline 4 & 1608 & 1659 & 1556 \\
\hline
\end{tabular}

TABLE II

FitTed PERMANENT DisPlaCEMENT ERROR OVER FIRST $30 \mathrm{~s}$ WITH $10 \%$ NOISE

\begin{tabular}{|r|r|r|r|}
\hline Storey & Mean Error & $\mathbf{9 0} \%$ Confidence Maximum & $\mathbf{9 0 \%}$ Confidence Minimum \\
\hline $\mathbf{1}$ & 0.05 & 7.23 & -5.90 \\
\hline $\mathbf{2}$ & 0.18 & 6.05 & -4.82 \\
\hline $\mathbf{3}$ & 0.21 & 5.53 & -4.85 \\
\hline $\mathbf{4}$ & 0.15 & 6.41 & -5.58 \\
\hline
\end{tabular}

was constructed for the simulated structure using the equivalent viscous damping approach assuming $2 \%$ damping in each mode as follows:

$$
C=\left[\begin{array}{cccc}
2.18 & -0.63 & -0.11 & -0.05 \\
-0.63 & 2.07 & -0.68 & 0.17 \\
-0.11 & -0.68 & 2.01 & -0.79 \\
-0.05 & -0.17 & -0.79 & 1.39
\end{array}\right]
$$

Each story was given a yield displacement $Y$ of $0.04 \mathrm{~m}$, shaping parameter $n$ of 2 , and a bilinear factor $\alpha=0.1$. These parameters were chosen to provide realistic nonlinear structural behavior for the fitting algorithm to identify. The simulated structure was subjected to the El-Centro earthquake record. The results for the identified stiffness and permanent displacement are shown in Tables I and II.

Both the stiffness and permanent displacement errors are identified accurately to within $8 \%$.

Note that it was also found that errors of even up to $100 \%$ in the damping matrix had a less than $5 \%$ effect on the identified stiffness and permanent displacement but for reasons of space these results are omitted. Hence, in simulation, the method of Fig. 2 is quite robust even with $10 \%$ noise added.

\section{B. Laboratory Test}

The structure used to test the algorithm in a real situation was a one-fifth scale four-story steel frame structure originally designed to demonstrate the dynamic response of multiple DF systems. [14]. The structure, shown in Fig. 3, features a two-bay frame in the longitudinal direction with one long span and one short span. The frame has overall dimensions of $2.1 \mathrm{~m} \times 2.1 \mathrm{~m}$ with a short-span length of $0.7 \mathrm{~m}$ and a long-span length of $1.4 \mathrm{~m}$. The first story has a height of $0.6 \mathrm{~m}$, while the second to fourth stories have a height of $0.5 \mathrm{~m}$. Replaceable plastic hinges are located at beam column joints and at the midpoints of the long-span beams.

A realistic and well-validated computational model of the structure has been developed for use with the time-history analysis package Ruaumoko [14], [15]. This computational model was used to estimate simplified four DF mass and damping matrices for use in the identification method of Fig. 2. However, importantly, note that precise damping matrix, as shown in simulation, is not critical for the algorithm of Fig. 2. The model [14] was also used to estimate the stiffness of each story using a pushover analysis, which can represent the "true" stiffness of the structure in Fig. 3. Hence, when applying Fig. 2 to measured experimental data from the structure, the identified stiffness can 


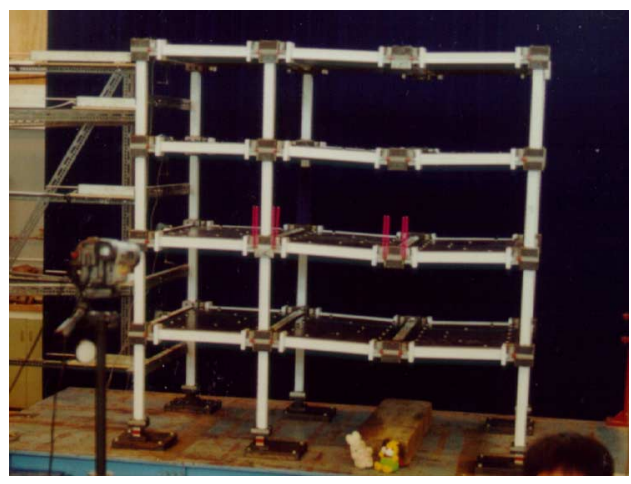

Fig. 3. Four-story nonlinear steel frame structure.

TABLE III

Pushover ANALysis Estimated Story StIFFnESSES

\begin{tabular}{|r|r|}
\hline Storey & Stiffness (kN/m) \\
\hline $\mathbf{1}$ & 672 \\
\hline $\mathbf{2}$ & 640 \\
\hline 3 & 601 \\
\hline $\mathbf{4}$ & 544 \\
\hline
\end{tabular}

be compared with the "true" stiffness to provide a further validation of the method. The following four DF diagonal mass matrix was calculated from the Ruaumoko model:

$$
M=\left[\begin{array}{cccc}
464 & 0 & 0 & 0 \\
0 & 464 & 0 & 0 \\
0 & 0 & 464 & 0 \\
0 & 0 & 0 & 461
\end{array}\right]
$$

Table III shows the story stiffnesses that were calculated by pushover analysis from the Ruaumoko model.

The damping matrix used in fitting the experimental structure was estimated by multiplying the simulation damping matrix (41) by 464 to scale to the correct mass, yielding

$$
C_{\mathrm{R}}=1 \times 10^{3}\left[\begin{array}{cccc}
1.01 & -0.29 & -0.05 & -0.03 \\
-0.29 & 0.96 & -0.32 & -0.08 \\
-0.05 & -0.32 & 0.93 & -0.37 \\
-0.03 & -0.08 & -0.37 & 0.64
\end{array}\right] \text {. }
$$

The structure was subjected to the El-Centro ground motion record, and structural acceleration and displacement data were measured at $1 \mathrm{kHz}$. No GPS was available so to approximately mimic these measurements, displacement was taken at $1 \mathrm{~Hz}$ with $10 \%$ random noise added. The parameters of $\Delta t_{k p}=2$ and $\Delta t_{z}=0.4$ are shown in Fig. 1.

Fig. 4 shows the fitted stiffness of the bottom story compared to that predicted by pushover analysis of a computational model constructed by [14]. Fig. 5 shows the fitted permanent displacement of the bottom story overlaid with the measured displacement. Note that the bottom story is chosen since the structure is first mode dominant and the bottom story therefore yields more than the higher story's.

Fig. 5 shows that the fitted final residual displacement matches the displacement response well. Also, the most significant yielding occurs at the same time as the largest peaks in displacement response as expected. Note also that the structure yields effectively twice, as shown in the figure, indicating that total absolute permanent deflection was reasonably captured.

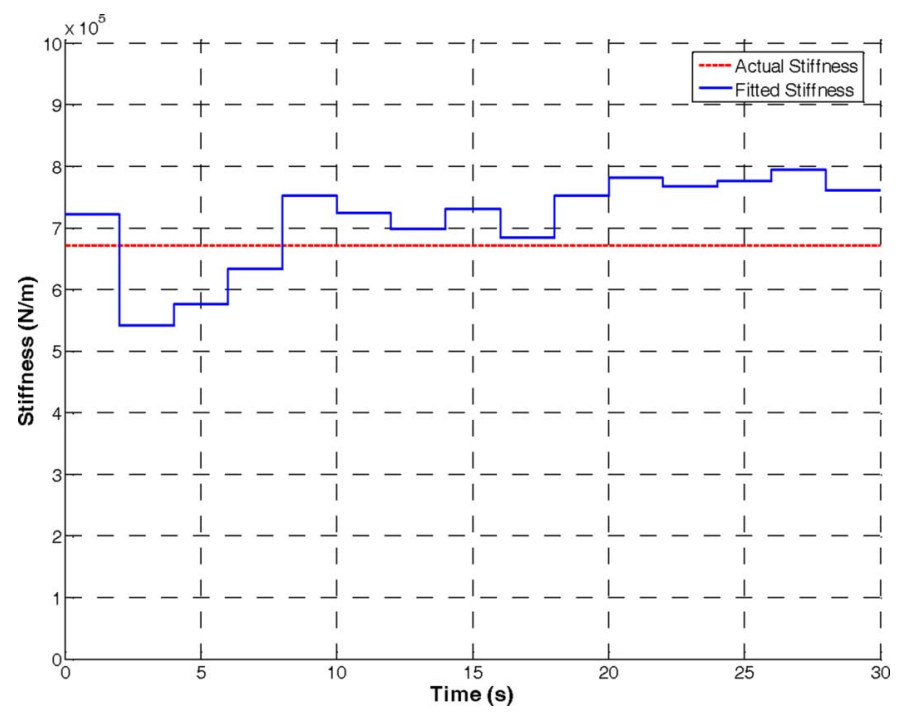

Fig. 4. Bottom story stiffness comparison for real structure.

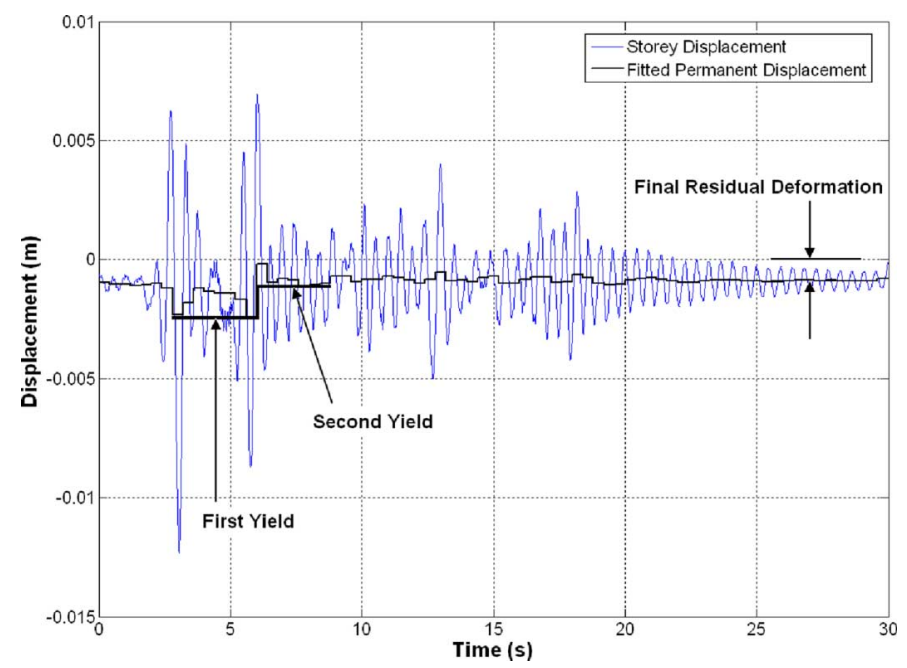

Fig. 5. Bottom story permanent displacement overlay for real structure.

The fitted permanent displacement is therefore reasonable in both behavior and magnitude. It can be seen in Fig. 4 that, as with the simulated results, the value of the fitted stiffness drops between 2 and $8 \mathrm{~s}$ where significant yielding occurs. This result likely occurs because the piecewise constant approximation to $\Delta Z(t)$ in (29) may no longer accurately model the hysteretic behavior over the interval, during this highly nonlinear period. Thus, a potentially better way may be to use a piecewise linear approximation, or more sophisticated model, but this is left for future work. However, this error does not reduce the algorithms ability to identify the overall stiffness. Table IV shows a summary of the fitted stiffnesses over the entire record where the predicted value is from the pushover analysis.

It is evident in Fig. 4 that the stiffness is most accurately identified during the period from 20 to $30 \mathrm{~s}$, where there is a reasonable magnitude of response but little yielding. Table $\mathrm{V}$ shows a summary of the fitted stiffnesses over this period.

The stiffness values in Table IV are within 3.5\% of the mean value for the first three stories; however, the values for the fourth story contain a maximum error of $14 \%$. Each story, $w$, is fitted using the sum of all equations of motion from $k=w, \ldots, \bar{N}$. 
TABLE IV

SUMMARY OF FITTED STIFFNESS FOR REAL STRUCTURE

\begin{tabular}{|r|r|r|r|r|}
\hline Storey & Mean (kN/m) & Max (kN/m) & Min (kN/m) & Predicted (kN/m) \\
\hline 1 & 713 & 795 & 541 & 672 \\
\hline 2 & 765 & 836 & 614 & 640 \\
\hline 3 & 662 & 724 & 548 & 601 \\
\hline 4 & 490 & 579 & 370 & 544 \\
\hline
\end{tabular}

TABLE V

SUMMARY OF FITTED STIFFNESS FROM 20-30 s

\begin{tabular}{|r|r|r|r|r|}
\hline Storey & Mean (kN/m) & Max (kN/m) & Min (kN/m) & Predicted (kN/m) \\
\hline 1 & 777 & 795 & 762 & 672 \\
\hline 2 & 810 & 836 & 791 & 640 \\
\hline 3 & 674 & 686 & 664 & 601 \\
\hline 4 & 532 & 576 & 458 & 544 \\
\hline
\end{tabular}

The reason for this greater error in the fourth story is that the parameters are fitted using measured data from only one story, as can be inferred from (31). Thus, with less data, the identification process is more susceptible to noise or sensor error. Other stories are fitted using measured data from multiple stories so are more accurately identified. Overall, the errors are within reasonable tolerances given variations in, for example, construction and material properties.

The fitted stiffnesses shown in Tables IV and V differ more significantly from those predicted by pushover analysis. There are many factors, which could contribute to these differences. Primarily, strain hardening of the plastic hinges due to the structure being repeatedly subjected to earthquake excitation. Also, [14] observed increases in flexural stiffness of structural members due to strain rate effects and the Bauschinger effect during high-amplitude motion. Finally, the pushover analysis model may also have limitations or other errors as it is a static test.

Another contributing factor to the differences between the identified and predicted stiffnesses is the modeling error. During fitting, the structure is assumed to be a four-DF shear building. However, in reality, the structure contains 51 structural members, which results in considerable geometric nonlinearity during high-amplitude motion. In addition, the structure undergoes appreciable flexural deformation during earthquake excitation, which is not accounted for in the fitted baseline shear building model.

As a further validation of the method of Fig. 2, the identified stiffness and permanent displacement in Figs. 4 and 6 corresponding to $\Delta t_{k p}=2$ and $\Delta t_{z}=0.4$ are used to resimulate the model of (1). A resimulation is also done for the parameters $\Delta t_{k p}=2$ and $\Delta t_{z}=0.2$ as a comparison. The errors in the simulated displacements are shown in Fig. 6.

The error for the time interval of 0.1 is on average less than 1 $\mathrm{mm}$ in displacement where as for the time interval of 0.4 is much higher around $4-5 \mathrm{~mm}$. These results are expected as the larger the interval where $\Delta Z(t)$ is constant over in Fig. 1, the less ability the model of (1) has to respond to the sudden nonlinear changes in dynamics. However, the longer fitting period of $0.4 \mathrm{~s}$ is more appropriate in this case, as major trends in permanent displacement are identified without undesirable oscillation in the solution (which happens as the period is reduced). Overall, a tradeoff between instantaneous accuracy and accurate longer term trend is presented in this case.

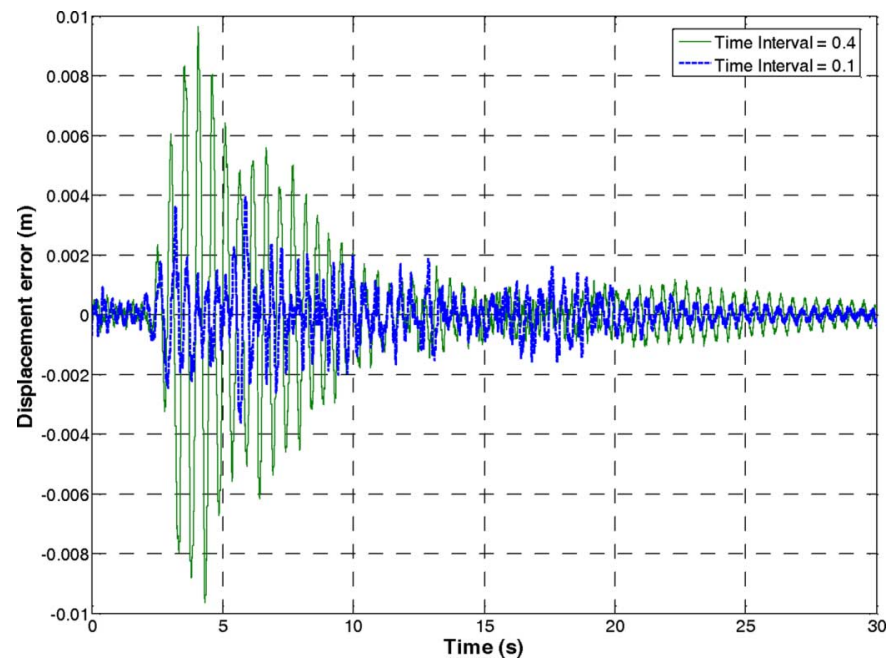

Fig. 6. Comparison of errors in resimulated first-story displacements.

\section{Application to SHM-Discussion}

The results of the estimated stiffness and permanent displacement in Figs. 4 and 5 are essentially equivalent to a real-time system identification of the four-story nonlinear steel frame structure of Fig. 3. Specifically, using MATLAB, identification of the stiffness and permanent displacement parameters in all four stories takes $\sim 0.17 \mathrm{~s}$ for each piecewise step of $2 \mathrm{~s}$ on a $3 \mathrm{GHz}$ Pentium 4 machine. In addition, the parameter identification at each step only relies on the previous history up to the current time point, since the displacement corrector function of (15) is cumulative. Coding in C typically gives at least an order of magnitude increase in computational speed. Thus, the main parameters of time-varying stiffness and permanent displacement of any given building could be readily provided by the algorithm in real time. Any environmental effects and/or operational condition changes would be recorded in terms of their effect on stiffness and permanent displacement. Furthermore, multiple damage scenarios are automatically handled as it is assumed that every floor is monitored using accelerometers and GPS. For example, if two floors are damaged, this situation will show up in the parameter identification as reduced stiffness in the two floors and/or permanent displacements. However, further work is required to validate the approach using actual GPS measured displacements.

\section{CONCLUSION}

The algorithm accurately identifies stiffness and permanent displacement in multiple DF systems. Since the algorithm effectively decouples the fitting process into separate optimizations for each story, it is easily generalized to higher DF. Simulated results, show that the stiffness of a particular story can be identified to within $\pm 3.5 \%$ with $90 \%$ confidence and permanent displacement can be identified to within $\pm 7.2 \%$ with $90 \%$ confidence, using $1 \mathrm{kHz}$ acceleration and $1 \mathrm{~Hz}$ displacement data subject to $10 \%$ uniformly distributed noise. The algorithm's accuracy could be improved in the long term by sampling ground motion and structural response at higher frequency and by fitting a piecewise linear permanent displacement, rather than the piecewise constant approach presented, which does not always accurately capture hysteretic response. In the short term, 
a simple moving average procedure could be used to identify permanent displacement more accurately.

When applied to measured data from a four-story steel frame structure, the algorithm identified a realistic permanent displacement and stiffness for each story. Discrepancies between the identified stiffness and a predicted stiffness calculated by pushover analysis of a previously constructed computational model are attributed to geometric nonlinearity, flexural deformation not accounted for by the baseline model, and strain hardening of connections from repeated testing.

In summary, this paper provides a highly efficient and accurate method for identifying linear stiffness and permanent displacement in multistory buildings under seismic loads. Identification of permanent deformation is novel in this field and can provide significant feedback to engineers as damage state and is also an ideal measure for the construction of probabilistic fragility functions. The overall method can be readily generalized to higher DF and is computationally efficient.

\section{REFERENCES}

[1] S. W. Doebling, C. R. Farrar, M. B. Prime, and D. W. Shevitz, Damage identification and health monitoring of structural and mechanical systems from changes in their vibration characteristics: A literature review Los Alamos Natl. Lab.,, 1996, Rep. LA-13070-MS.

[2] G. Konstantinidis, P. D. Wilcox, and B. W. Drinkwater, "An investigation into the temperature stability of a guided wave structural health monitoring system using permanently attached sensors," IEEE Sensors J., vol. 7, pp. 905-912, May 2007.

[3] J. E. Doherty, "Non-destructive evaluation in Handbook on Experimental Mechanics," in , A. S. Kobayashi, Ed. Bethel, CT: Society for Experimental Mechanics, Inc, 1997, ch. 12.

[4] J. G. Chase, K. L. Hwang, L. R. Barroso, and J. B. Mander, "A simple LMS-based approach to the structural health monitoring benchmark problem," J. Earthquake Eng. Struct. Dyn., vol. 34, no. 6, pp. 575-594, 2004.

[5] C. R. Farrar and S. W. Doebling, "An overview of modal-based damage identification methods," in EUROMECH 365 Int. Workshop: DAMAS 1997, Sheffield, U.K., 1997, Structural Damage Assessment Using Advanced Signal Processing Procedures [Online]. Available: http://mesl. ucsd.edu/gupta/SHM/Reading/damas_overview.pdf

[6] O. Buyukozturk and T. Yu, "Structural health monitoring and seismic impact assessment," in Proc. 5th Natl. Conf. Earthquake Eng., Istanbul, Turkey, May 26-30, 2003, pp. 1-21.

[7] D. H. Kim and M. Q. Feng, "Real-time structural health monitoring using a novel fiber-optic accelerometer system," IEEE Sensors J., vol. 7, pp. 536-543, 2007.

[8] M. D. Kohler, P. M. Davis, and E. Safak, "Earthquake and ambient vibration monitoring of the steel frame UCLA factor building," Earthquake Spectra, vol. 21, no. 3, pp. 715-715, 2005.

[9] E. Safak and K. Hudnut, "Real-time structural monitoring and damage detection by acceleration and GPS sensors," in 8th US Natl. Conf. Earthquake Eng., San Francisco, CA, 2006 [Online]. Available: http://www.rotational-seismology.org/library/publications/publicationpresentedpaper.2006-07-19.8888101901

[10] P. A. Psimoulis and S. C. Stiros, "Experimental assessment of the accuracy of GPS and RTS for the determination of the parameters of oscillation of major structures," Comput.-Aided Civil Infrastructure Eng., vol. 23, pp. 389-402, 2008.

[11] W. S. Chan, Y. L. Xu, X. L. Ding, Y. L. Xiong, and W. J. Dai, "Assessment of dynamic measurement accuracy of GPS in three directions," $J$. Surveying Eng., ASCE, vol. 132, no. 3, pp. 108-117, 2006.

[12] Y.-K. Wen, "Method for random vibration of hysteretic systems," $J$. Eng. Mech. Div., ASCE, vol. 102, no. 2, pp. 249-263, 1976.

[13] M. V. Sivaselvan and A. M. Reinhorn, "Hysteretic models for deteriorating inelastic structures," J. Eng. Struct., vol. 126, pp. 633-640, 2000.

[14] G. C. Kao, "Design and shaking table tests of a four-storey miniature structure built with replaceable plastic hinges," Masters of Civil Engineering Thesis, Univ. Canterbury, , New Zealand, 1998.
[15] M. E. Rodriguez, J. I. Restrepo, and J. J. Blandon, "Shaking table tests of a four-story miniature steel building-Model validation," Earthquake Spectra, vol. 22, no. 3, pp. 755-780, 2006.

Christopher E. Hann received the B.Sc. (with first class honors) and the Ph.D. degrees, both in mathematics, from the University of Canterbury, Christchurch, New Zealand, in 1996 and 2001, respectively.

He then worked as a Teaching Fellow in the Department of Mathematics until 2003, and received a Postdoctorate position at the Department of Mechanical Engineering, University of Canterbury, in 2004. From 2007 onward, he has been a Sir Charles Hercus Health Research Fellow and a Senior Research Associate. He has authored or coauthored more than 180 refereed journal and conference papers, is an inventor on several patents, and is on the editorial board for an Open Bentham journal in medical informatics. His research interests include computational and physiological modeling for biomedical systems, model-based diagnostic and therapeutics for critical care, physiological parameter identification, and the application of image processing and computer vision in biomedicine.

Ishan Singh-Levett received the B.E. degree (with honors) in mechanical engineering, in 2005, and the Master's of Engineering degree, in 2006, both from the University of Canterbury, Christchurch, New Zealand.

Currently, he is a Mechanical Engineer for HGL Dynamics Ltd., Godalming, U.K.

Bruce L. Deam is the Leicester Steven EQC Lecturer in Earthquake Engineering at the University of Canterbury, Christchurch, New Zealand. He is a Structural Engineer who specializes in the behavior and design of houses and large timber structures subjected to earthquake ground motion. He has authored or coauthored more than 50 papers. His recent research interests and teaching responsibilities include structural systems, structural dynamics, and numerical methods.

Dr. Deam is a Fellow of the New Zealand Society for Earthquake Engineering and an Assistant Editor for their quarterly journal.

John B. Mander received the NZCE degree in civil engineering from the Christchurch Polytechnic, Christchurch, New Zealand, in 1976, the B.E. degree (with first class honors) in civil engineering, in 1979, and the Ph.D. degree in civil engineering, in 1984.

From 1983 to 1986, he was a Systems Engineer at the NIMT Railway Electrification Project Office and in 1987 he was a Deputy Group Manager and Strategic Planning Manager for New Zealand Railways Corporation. He was an Assistant Professor at the Department of Civil Engineering, State University of New York at Buffalo, from 1988 to 1995, an Associate Professor in the same institute from 1995 to 2000, and a Professor and the Chair of Structural Engineering at the Department of Civil Engineering, University of Canterbury, from 2000 to 2007. From 2007 onward, he is the Zachry Professor in Design and Construction Integration at the Zachry Department of Civil Engineering, Texas A\&M University, College Station. He has authored or coauthored more than 150 refereed journal and conference papers and 2 patents, in U.S. and France, and is an Associate Editor of the Journal of Structural Engineering.

J. Geoffrey Chase received the B.S. degree in mechanical engineering from Case Western Reserve University, Cleveland, OH, in 1986, and the M.S. and Ph.D. degrees form Stanford University, Stanford, CA, in 1991 and 1996, respectively, in mechanical and civil engineering.

He spent six years working for General Motors between degrees and a further five years consulting in Silicon Valley, including positions at Xerox PARC, GN ReSound, and Infineon Technologies AG. He started at the University of Canterbury in 2000 . He has authored or coauthored more than 380 refereed journal and conference papers and is an inventor on over ten patents. His research interests include biomedical systems and devices, structural dynamics and vibrations, dynamic systems modeling, automatic control, microelectromechanical systems and microfabrication, and embedded systems.

Dr. Chase is on the editorial board of three journals in bioengineering and medicine and is a member of IFAC TC 8.2 on Biological and Medical Systems. 